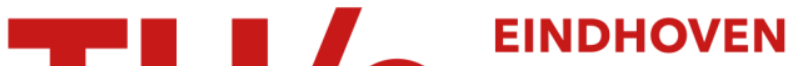 UNIVERSITY OF TECHNOLOGY
}

\section{A compact high order finite volume scheme for advection- diffusion-reaction equations}

\section{Citation for published version (APA):}

Anthonissen, M. J. H., \& Thije Boonkkamp, ten, J. H. M. (2009). A compact high order finite volume scheme for advection-diffusion-reaction equations. (CASA-report; Vol. 0924). Technische Universiteit Eindhoven.

\section{Document status and date:}

Published: 01/01/2009

\section{Document Version:}

Publisher's PDF, also known as Version of Record (includes final page, issue and volume numbers)

\section{Please check the document version of this publication:}

- A submitted manuscript is the version of the article upon submission and before peer-review. There can be important differences between the submitted version and the official published version of record. People interested in the research are advised to contact the author for the final version of the publication, or visit the $\mathrm{DOI}$ to the publisher's website.

- The final author version and the galley proof are versions of the publication after peer review.

- The final published version features the final layout of the paper including the volume, issue and page numbers.

Link to publication

\section{General rights}

Copyright and moral rights for the publications made accessible in the public portal are retained by the authors and/or other copyright owners and it is a condition of accessing publications that users recognise and abide by the legal requirements associated with these rights.

- Users may download and print one copy of any publication from the public portal for the purpose of private study or research.

- You may not further distribute the material or use it for any profit-making activity or commercial gain

- You may freely distribute the URL identifying the publication in the public portal.

If the publication is distributed under the terms of Article 25fa of the Dutch Copyright Act, indicated by the "Taverne" license above, please follow below link for the End User Agreement:

www.tue.nl/taverne

Take down policy

If you believe that this document breaches copyright please contact us at:

openaccess@tue.nl

providing details and we will investigate your claim. 


\section{EINDHOVEN UNIVERSITY OF TECHNOLOGY}

Department of Mathematics and Computer Science

\section{CASA-Report 09-24}

August 2009

A compact high order finite volume scheme for advection-diffusion-reaction equations

by

M.J.H. Anthonissen, J.H.M. ten Thije Boonkkamp

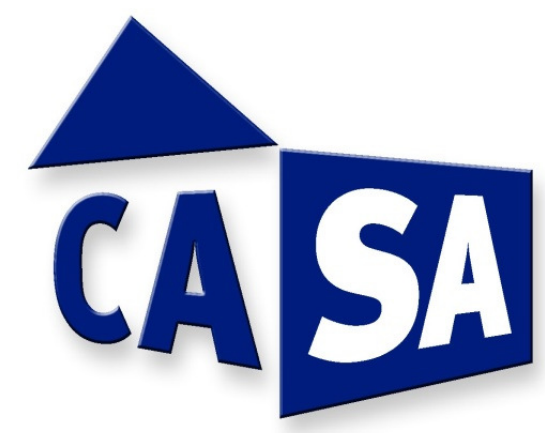

Centre for Analysis, Scientific computing and Applications

Department of Mathematics and Computer Science

Eindhoven University of Technology

P.O. Box 513

5600 MB Eindhoven, The Netherlands

ISSN: 0926-4507 


\title{
A compact high order finite volume scheme for advection-diffusion-reaction equations
}

\author{
M.J.H. Anthonissen and J.H.M. ten Thije Boonkkamp \\ Department of Mathematics and Computer Science, Eindhoven University of Technology, \\ P.O. Box 513, 5600 MB Eindhoven, The Netherlands
}

\begin{abstract}
We present a new integral representation for the flux of the advection-diffusion-reaction equation, which is based on the solution of a local boundary value problem for the entire equation, including the source term. The flux therefore consists of two parts, corresponding to the homogeneous and particular solution of the boundary value problem. Applying Gauss-Legendre quadrature rules to the integral representation gives the high order finite volume complete flux scheme, which is fourth order accurate for both diffusion dominated and advection dominated flow.
\end{abstract}

Keywords: Advection-diffusion-reaction equation, flux, finite volume method, integral representation of the flux, numerical flux PACS: 02.30.Jr, 02.60.-x, 02.60.Cb, 02.60.Jh, 02.60.Lj, 02.70.Bf

\section{INTRODUCTION}

Conservation laws are ubiquitous in continuum physics, they occur in disciplines like fluid mechanics, combustion theory, plasma physics, semiconductor physics etc. These conservation laws are often of advection-diffusion-reaction type, describing the interplay between different processes such as advection or drift, diffusion or conduction and (chemical) reaction or recombination/generation. Examples are the conservation equations for reacting flow [7, 12] or the drift-diffusion equations for semiconductor devices [3,5].

Their numerical solution requires at least an adequate (space) discretization. There are many (classes of) methods available, such as finite element, finite difference, finite volume or spectral methods. We restrict ourselves to finite volume methods; for a detailed account see e.g. [6,11,1]. Finite volume methods are based on the integral formulation, i.e., the conservation law is integrated over a disjunct set of control volumes covering the domain. The resulting discrete conservation law involves fluxes at the interfaces of the control volumes, which need to be approximated.

Our objective in this paper is to present new expressions for the flux, which will subsequently be used to derive numerical flux approximations. We restrict ourselves to steady one-dimensional conservation equations. For the flux approximation we use Gauss-Legendre quadrature, which results in a fourth order accurate discretization scheme. The numerical flux will only depend on neighboring values of the unknown, resulting in a three-point scheme. The linear system that needs to be solved is therefore tridiagonal. This compactness is a clear advantage over high resolution schemes based on flux/slope limiters $[4,11]$ or $(\mathrm{W})$ ENO reconstruction [8]. Our scheme is inspired by two papers by Thiart $[9,10]$.

\section{FINITE VOLUME DISCRETIZATION}

In this section we outline the finite volume method (FVM) for a generic conservation law of advection-diffusionreaction type. So, consider the following conservation law defined on the interval $(a, b)$

$$
\left(m \varphi-\varepsilon \varphi^{\prime}\right)^{\prime}=s,
$$

where $m$ is the mass flux, $\varepsilon \geq \varepsilon_{\min }>0$ a diffusion/conduction coefficient and $s$ a (chemical) source term. The unknown $\varphi$ can be, e.g., the temperature or the concentration of a species in a reacting flow. The parameters $\varepsilon$ and $s$ are usually (complicated) functions of the unknown $\varphi$, however, for the sake of discretization we will consider these as given functions of the spatial coordinate $x$. The mass flux $m$ generally has to be computed from the flow equations corresponding to (1), but in this paper it is assumed to be a given function of $x$ as well. Equations of this type arise, e.g., in combustion theory [7]. 
In the FVM, we define a spatial grid $\left\{x_{j}\right\}$ where the variable $\varphi$ has to be approximated and we cover the interval $(a, b)$ with a finite number of disjunct intervals (control volumes) $I_{j}$. In this paper we choose the cell-centered approach [11], i.e., we choose the grid point $x_{j}$ in the center of the $j$ th interval $I_{j}$. Consequently we have $I_{j}:=\left(x_{j-1 / 2}, x_{j+1 / 2}\right)$ with $x_{j+1 / 2}:=\left(x_{j}+x_{j+1}\right) / 2$. For ease of presentation, we shall use a uniform grid. Denoting the number of grid points by $N$, we have $x_{j}=a+j \Delta x(j=0,1, \ldots, N-1)$ with $\Delta x:=(b-a) /(N-1)$ the length of each control volume.

Associated with equation (1) we introduce the flux $f:=m \varphi-\varepsilon \varphi^{\prime}$. Equation (1) then reduces to $f^{\prime}=s$. Integrating this equation over the control volume $I_{j}, j=1,2, \ldots, N-2$, we obtain the discrete conservation law

$$
F_{j+1 / 2}-F_{j-1 / 2}=Q_{j}
$$

where $F_{j+1 / 2}$ is the numerical flux approximating $f$ at $x=x_{j+1 / 2}$ and where $Q_{j}$ is a quadrature rule for the integral of $s$ over $I_{j}$. We shall present a numerical flux $F_{j+1 / 2}$ that depends on $\varphi$ in the neighboring grid points $x_{j}$ and $x_{j+1}$ only. Moreover, this dependence will be linear. This means that we are looking for an expression of the form

$$
F_{j+1 / 2}=\alpha_{j+1 / 2} \varphi_{j}+\beta_{j+1 / 2} \varphi_{j+1}+\gamma_{j+1 / 2}(s),
$$

where the coefficients $\alpha_{j+1 / 2}$ and $\beta_{j+1 / 2}$ only depend on $m$ and $\varepsilon$, and where $\gamma_{j+1 / 2}$ depends on $m, \varepsilon$ and $s$. Substitution of this expression in the discrete conservation law (2) leads to a tridiagonal linear system for the vector of unknowns $\varphi=\left(\varphi_{1}, \varphi_{2}, \ldots, \varphi_{N-2}\right)^{T}$.

\section{INTEGRAL REPRESENTATION FOR THE FLUX}

We first find an integral representation for the flux. Our numerical flux will readily follow from this representation. The derivation is a modification of the theory in [2]. In order to find the flux $f_{j+1 / 2}$ at the eastern cell edge $x_{j+1 / 2}$ located between the grid points $x_{j}$ and $x_{j+1}$ we consider the boundary value problem (BVP) $\left(m \varphi-\varepsilon \varphi^{\prime}\right)^{\prime}=s$ for the unknown $\varphi$ on $\left(x_{j}, x_{j+1}\right)$ with Dirichlet boundary conditions $\varphi\left(x_{j}\right)=\varphi_{j}, \varphi\left(x_{j+1}\right)=\varphi_{j+1}$. It is convenient to define the variables $\lambda, P, \Lambda$ and $S$ for $x \in\left(x_{j}, x_{j+1}\right)$ by

$$
\lambda:=\frac{m}{\varepsilon}, \quad P:=\lambda \Delta x, \quad \Lambda(x):=\int_{x_{j+1 / 2}}^{x} \lambda(\xi) \mathrm{d} \xi, \quad S(x):=\int_{x_{j+1 / 2}}^{x} s(\xi) \mathrm{d} \xi .
$$

Here, $\lambda$ is the ratio of advection and diffusion, $P$ and $\Lambda$ are the Peclet function and Peclet integral and $S$ is the integral of the source term s. $P$ and $\Lambda$ generalize the well-known Peclet number [11]. Integrating the differential equation from $x_{j+1 / 2}$ to $x$ we get the integral balance $f-f_{j+1 / 2}=S$. Using the definition of $\Lambda$ in (4), it is clear that the flux can be rewritten as $f=-\varepsilon\left(\varphi \mathrm{e}^{-\Lambda}\right)^{\prime} \mathrm{e}^{\Lambda}$. Substituting this into the integral balance and integrating from $x_{j}$ to $x_{j+1}$, we obtain the following expression for the flux $f_{j+1 / 2}$ :

$$
f_{j+1 / 2}=-\frac{\mathrm{e}^{-\Lambda_{j+1}} \varphi_{j+1}-\mathrm{e}^{-\Lambda_{j}} \varphi_{j}}{\int_{x_{j}}^{x_{j+1}} \varepsilon^{-1} \mathrm{e}^{-\Lambda} \mathrm{d} x}-\frac{\int_{x_{j}}^{x_{j+1}} \varepsilon^{-1} \mathrm{e}^{-\Lambda} S \mathrm{~d} x}{\int_{x_{j}}^{x_{j+1}} \varepsilon^{-1} \mathrm{e}^{-\Lambda} \mathrm{d} x},
$$

where $\Lambda_{j}=\Lambda\left(x_{j}\right)$, etc. We set $C_{j}:=1 / \int_{x_{j}}^{x_{j+1}} \varepsilon^{-1} \mathrm{e}^{-\Lambda} d x$, and define the homogeneous and inhomogeneous fluxes as

$$
f_{j+1 / 2}^{(\mathrm{h})}:=C_{j} \mathrm{e}^{-\Lambda_{j}} \varphi_{j}-C_{j} \mathrm{e}^{-\Lambda_{j+1}} \varphi_{j+1}, \quad f_{j+1 / 2}^{(\mathrm{i})}:=-C_{j} \int_{x_{j}}^{x_{j+1}} \varepsilon^{-1} \mathrm{e}^{-\Lambda} S \mathrm{~d} x .
$$

Note that these fluxes correspond to the homogeneous and particular solution of the BVP, respectively. Moreover, only the homogeneous flux depends on $\varphi$. Choosing a suitable quadrature rule for all integrals appearing in the coefficients $C_{j} \mathrm{e}^{-\Lambda_{j}}$ and $-C_{j} \mathrm{e}^{-\Lambda_{j+1}}$ leads to the first two terms in the right hand side of (3). The third term will follow from the inhomogeneous flux that we consider next.

Substituting the expression for $S$ in (6), changing the order of integration and applying the linear transformation $\sigma:=\left(x-x_{j}\right) / \Delta x$, we find the following representation for the inhomogeneous flux

$$
f_{j+1 / 2}^{(\mathrm{i})}=\Delta x \int_{0}^{1} G(\sigma) s\left(x_{j}+\sigma \Delta x\right) \mathrm{d} \sigma, \quad G(\sigma)= \begin{cases}C_{j} \Delta x \int_{0}^{\sigma} \varepsilon^{-1} \mathrm{e}^{-\Lambda} \mathrm{d} \eta, & \text { for } 0 \leq \sigma \leq 1 / 2, \\ -C_{j} \Delta x \int_{\sigma}^{1} \varepsilon^{-1} \mathrm{e}^{-\Lambda} \mathrm{d} \eta, & \text { for } 1 / 2<\sigma \leq 1,\end{cases}
$$


in which $G$ is the normalized Green's function for the flux. Note that $G$ relates the flux to the source term and is different from the usual Green's function, which relates the solution to the source term [6]. $G$ is discontinuous at $\sigma=\frac{1}{2}$ with jump $G\left(\frac{1}{2}^{-}\right)-G\left(\frac{1}{2}^{+}\right)=1$. The value $\sigma=\frac{1}{2}$ corresponds with $x=x_{j+1 / 2}$. For the special case of constant $m$ and $\varepsilon$ the Green's function reduces to

$$
G(\sigma ; P)= \begin{cases}\left(1-\mathrm{e}^{-P \sigma}\right) /\left(1-\mathrm{e}^{-P}\right), & \text { for } 0 \leq \sigma \leq \frac{1}{2} \\ -\left(1-\mathrm{e}^{P(1-\sigma)}\right) /\left(1-\mathrm{e}^{P}\right), & \text { for } \frac{1}{2}<\sigma \leq 1\end{cases}
$$

Note that we use the notation $G=G(\sigma ; P)$ to denote the dependence on the Peclet number $P$. $G$ satisfies the symmetry property $G(\sigma ; P)=-G(1-\sigma ;-P)$. We shall base our numerical approximation of the inhomogeneous flux on (7) with the Green's function $G$ replaced by (8), so we use (8) even for nonconstant $P$.

\section{DERIVATION OF THE NUMERICAL FLUX}

In this section we propose quadrature rules for all integrals that appear in the expressions we have derived for the homogeneous and inhomogeneous fluxes. Since we would like the finite volume scheme to be fourth order accurate, we choose the standard 2-point Gauss-Legendre approximation, viz.

$$
\int_{a}^{b} g(x) \mathrm{d} x \approx \frac{b-a}{2}\left(g\left(\frac{a+b}{2}-\frac{b-a}{2 \sqrt{3}}\right)+g\left(\frac{a+b}{2}+\frac{b-a}{2 \sqrt{3}}\right)\right)=: \operatorname{GL}(g, a, b) .
$$

We start with the homogeneous flux. We use (9) to approximate $\Lambda(x)$ with $\operatorname{GL}\left(\lambda, x_{j+1 / 2}, x\right)$. Next we apply the quadrature rule again to find $C_{j} \approx 1 / \mathrm{GL}\left(\varepsilon^{-1} \mathrm{e}^{-\mathrm{GL}\left(\lambda, x_{j+1 / 2}, x\right)}, x_{j}, x_{j+1}\right)$. This provides us with a fourth order accurate approximation for the homogeneous flux, cf. (3),

$$
f_{j+1 / 2}^{(\mathrm{h})}=C_{j} \mathrm{e}^{-\Lambda_{j}} \varphi_{j}-C_{j} \mathrm{e}^{-\Lambda_{j+1}} \varphi_{j+1} \approx \alpha_{j+1 / 2} \varphi_{j}+\beta_{j+1 / 2} \varphi_{j+1},
$$

if we define

$$
\alpha_{j+1 / 2}:=\frac{\mathrm{e}^{-\mathrm{GL}\left(\lambda, x_{j+1 / 2}, x_{j}\right)}}{\mathrm{GL}\left(\varepsilon^{-1} \mathrm{e}^{-\mathrm{GL}\left(\lambda, x_{j+1 / 2}, x\right)}, x_{j}, x_{j+1}\right)}, \quad \beta_{j+1 / 2}:=-\frac{\mathrm{e}^{-\mathrm{GL}\left(\lambda, x_{j+1 / 2}, x_{j+1}\right)}}{\operatorname{GL}\left(\varepsilon^{-1} \mathrm{e}^{-\mathrm{GL}\left(\lambda, x_{j+1 / 2}, x^{\prime}\right.}, x_{j}, x_{j+1}\right)} .
$$

Next we consider the inhomogeneous flux. Use (7) and split the integral in the part from 0 to $1 / 2$ and the part from $1 / 2$ to 1 . On the first and second interval we need to integrate

$$
f_{1}(\sigma):=\frac{1-\mathrm{e}^{-P\left(x_{j}+\sigma \Delta x\right) \sigma}}{1-\mathrm{e}^{-P\left(x_{j}+\sigma \Delta x\right)}} \cdot s\left(x_{j}+\sigma \Delta x\right), \quad f_{2}(\sigma):=-\frac{1-\mathrm{e}^{P\left(x_{j}+\sigma \Delta x\right)(1-\sigma)}}{1-\mathrm{e}^{P\left(x_{j}+\sigma \Delta x\right)}} \cdot s\left(x_{j}+\sigma \Delta x\right),
$$

respectively. We use once more the Gauss-Legendre quadrature rule:

$$
f_{j+1 / 2}^{(\mathrm{i})} \approx\left(\mathrm{GL}\left(f_{1}, 0,1 / 2\right)+\mathrm{GL}\left(f_{2}, 1 / 2,1\right)\right) \Delta x=: \gamma_{j+1 / 2}(s) .
$$

\section{THE HIGH ORDER FINITE VOLUME-COMPLETE FLUX SCHEME}

The numerical flux at the eastern cell interface $x_{j+1 / 2}$ of the control volume $I_{j}$ can be written as in (3), where the coefficients $\alpha_{j+1 / 2}, \beta_{j+1 / 2}$ and the function $\gamma_{j+1 / 2}$ are as defined in the previous section. A similar expression holds for the numerical flux $F_{j-1 / 2}$ at the western cell interface $x_{j-1 / 2}$. Substituting these in the discrete conservation law (2) and using the Gauss-Legendre rule for $Q_{j}$, we find

$$
\beta_{j+1 / 2} \varphi_{j+1}+\left(\alpha_{j+1 / 2}-\beta_{j-1 / 2}\right) \varphi_{j}-\alpha_{j-1 / 2} \varphi_{j-1}=\mathrm{GL}\left(s, x_{j-1 / 2}, x_{j+1 / 2}\right)-\gamma_{j+1 / 2}(s)+\gamma_{j-1 / 2}(s),
$$

which we shall refer to as the high order finite volume-complete flux (HOCF) scheme. The scheme has a three-point coupling for $\varphi$, resulting in the following linear system $A \varphi=b$, where $\varphi$ is the vector of unknowns and where the vector $b$ contains the source term contributions and boundary data. The matrix $A$ is tridiagonal. 


\section{NUMERICAL RESULTS}

In this section we apply the central difference (CD) and the high order complete flux (HOCF) schemes to a model problem to assess their (order of) accuracy. We consider both diffusion-dominated and advection-dominated flow. We solve the BVP $\left(m \varphi-\varepsilon \varphi^{\prime}\right)^{\prime}=s$ on $(0,1)$, with Dirichlet boundary conditions $\varphi(0)=0, \varphi(1)=1$, and with mass flux $m(x)=1-b \sin (\pi x)$ and source term $s$ chosen such that the exact solution is given by $\varphi(x)=a(\sin (\alpha \pi x)-\sin (\alpha \pi))+$ $\frac{\mathrm{e}^{(x-1) / \varepsilon}-\mathrm{e}^{-1 / \varepsilon}}{1-\mathrm{e}^{-1 / \varepsilon}}$, see [11]. Note that for $0<\varepsilon \ll 1$ the solution has a thin boundary layer of width $\varepsilon$ near $x=1$. We take the following parameter values: $a=1, b=0.95, \alpha=3$ and $\varepsilon=1$ (dominant diffusion) or $\varepsilon=1 / 100$ (dominant advection). Let $h=\Delta x=1 /(N-1)$ be the grid size, with $N$ the number of grid points. To determine the accuracy of a numerical solution we compute the error $e^{h}:=\left\|\varphi-\varphi^{*}\right\|_{\infty}$, where $\varphi^{*}$ denotes the exact solution restricted to the grid.

Table 1 shows $e^{h}$ and the reduction factors $e^{h} / e^{h / 2}$ for both schemes. Clearly, $e^{h} / e^{h / 2} \rightarrow 4$ for $h \rightarrow 0$ for the CD scheme, and consequently, it displays second order convergence behaviour for $h \rightarrow 0$. For the new high order scheme $e^{h} / e^{h / 2} \rightarrow 16$, so it is indeed fourth order accurate for both dominant diffusion and dominant advection. Note that the accuracy of the HOCF scheme is vastly superior to CD on identical grids.

TABLE 1. Errors and error quotients.

\begin{tabular}{ccccccccc}
\hline \multicolumn{3}{c}{$\varepsilon=1$} & \multicolumn{3}{c}{$\varepsilon=1 / 100$} \\
\hline \multicolumn{3}{c}{$\mathrm{CD}$} & \multicolumn{2}{c}{ HOCF } & \multicolumn{2}{c}{ CD } & \multicolumn{2}{c}{ HOCF } \\
\hline$h^{-1}$ & $e^{h}$ & $e^{h} / e^{h / 2}$ & $e^{h}$ & $e^{h} / e^{h / 2}$ & $e^{h}$ & $e^{h} / e^{h / 2}$ & $e^{h}$ & $e^{h} / e^{h / 2}$ \\
10 & $7.977 \cdot 10^{-02}$ & 4.10 & $1.944 \cdot 10^{-04}$ & 16.21 & $5.321 \cdot 10^{-01}$ & 1.34 & $1.621 \cdot 10^{-01}$ & 7.93 \\
20 & $1.944 \cdot 10^{-02}$ & 3.99 & $1.199 \cdot 10^{-05}$ & 15.88 & $3.983 \cdot 10^{-01}$ & 2.14 & $2.043 \cdot 10^{-02}$ & 10.17 \\
40 & $4.875 \cdot 10^{-03}$ & 4.01 & $7.549 \cdot 10^{-07}$ & 16.03 & $1.863 \cdot 10^{-01}$ & 3.36 & $2.009 \cdot 10^{-03}$ & 13.90 \\
80 & $1.217 \cdot 10^{-03}$ & 4.00 & $4.708 \cdot 10^{-08}$ & 15.99 & $5.546 \cdot 10^{-02}$ & 4.56 & $1.445 \cdot 10^{-04}$ & 15.44 \\
160 & $3.045 \cdot 10^{-04}$ & 4.00 & $2.944 \cdot 10^{-09}$ & 16.01 & $1.217 \cdot 10^{-02}$ & 3.99 & $9.364 \cdot 10^{-06}$ & 15.85 \\
320 & $7.611 \cdot 10^{-05}$ & 4.00 & $1.839 \cdot 10^{-10}$ & 15.92 & $3.047 \cdot 10^{-03}$ & 4.03 & $5.907 \cdot 10^{-07}$ & 15.96 \\
640 & $1.903 \cdot 10^{-05}$ & & $1.155 \cdot 10^{-11}$ & & $7.557 \cdot 10^{-04}$ & & $3.701 \cdot 10^{-08}$ & \\
\hline
\end{tabular}

\section{CONCLUSIONS}

We have derived an integral representation for the flux of the advection-diffusion-reaction equation from a local BVP for the entire equation, including the source term. As a consequence, the flux consists of two parts, i.e., a homogeneous and an inhomogeneous part, corresponding to the homogeneous and particular solution of the BVP, respectively. Thus, the inhomogeneous part takes into account the effect of the source term on the flux. An alternative representation of the inhomogeneous flux in terms of a Green's function is given. The integral representation is the basis for the numerical flux approximation. We apply the 2-point Gauss-Legendre quadrature rule to all integrals involved, resulting in a fourth order accurate discretization, uniformly in the (local) Peclet numbers. Moreover, the discretization leads to a compact, tridiagonal system, which can be solved very efficiently. This feature is clearly an advantage over high resolution methods based on flux/slope limiters or (W)ENO reconstruction. Numerical results confirm that our method is fourth order accurate indeed.

\section{REFERENCES}

1. R. Eymard, T. Gallouët and R. Herbin, Finite volume methods, in: P.G. Ciarlet and J.L. Lions (eds.), Handbook of Numerical Analysis, Volume VII, North-Holland, Amsterdam, 2000, pp. 713-1020.

2. B. van 't Hof, J.H.M. ten Thije Boonkkamp and R.M.M. Mattheij, Discretization of the stationary convection-diffusion-reaction equation, Numer. Meth. for Part. Diff. Eq. 14, 607-625, 1998.

3. J.W. Jerome, Analysis of Charge transport, Springer, Berlin, 1996.

4. Randell J. LeVeque, Finite Volume Methods for Hyperbolic Problems, Cambridge Texts in Applied Mathematics, Cambridge University Press, Cambridge, 2002.

5. P.A. Markowich, C.A. Ringerhofer and C. Schmeiser, Semiconductor Equations, Springer, Vienna, 1990.

6. K.W. Morton, Numerical Solution of Convection-Diffusion Problems, Applied Mathematics and Mathematical Computation 12, Chapman \& Hall, London, 1996.

7. T. Poinsot and D. Veynante, Theoretical and Numerical Combustion, Second Edition, Edwards, Philadelphia, 2005. 
8. Chi-Wang Shu, Essentially non-oscillatory and weighted essentially non-oscillatory schemes for hyperbolic conservation laws, in: A. Quarteroni (ed.), Advanced Numerical Approximation of Nonlinear Hyperbolic Equations, Lecture Notes in Mathematics 1697, Springer, Berlin, 1998, pp. 325-432.

9. G.D. Thiart, Finite difference scheme for the numerical solution of fluid flow and heat transfer problems on nonstaggered grids, Numer. Heat Transfer, Part B 17, 41-62, 1990

10. G.D. Thiart, Improved finite-difference scheme for the solution of convection-diffusion problems with the SIMPLEN algorithm, Numer. Heat Transfer, Part B 18, 81-95, 1990.

11. P. Wesseling, Principles of Computational Fluid Dynamics, Springer Series in Computational Mathematics 29, Springer, Berlin, 2000.

12. Forman A. Williams, Combustion Theory, Second Edition, Addison-Wesley, Redwood City, 1985. 
PREVIOUS PUBLICATIONS IN THIS SERIES:

\begin{tabular}{|c|c|c|c|}
\hline Number & Author(s) & Title & Month \\
\hline $09-20$ & $\begin{array}{l}\text { K. Mohaghegh } \\
\text { R. Pulch } \\
\text { M. Striebel } \\
\text { E.J.W. ter Maten }\end{array}$ & $\begin{array}{l}\text { Model order reduction for } \\
\text { semi-explicit systems of } \\
\text { differential algebraic } \\
\text { equations }\end{array}$ & June '09 \\
\hline $09-21$ & $\begin{array}{l}\text { A. Verhoeven } \\
\text { M. Striebel } \\
\text { E.J.W. ter Maten }\end{array}$ & $\begin{array}{l}\text { Model order reduction for } \\
\text { nonlinear IC models with } \\
\text { POD }\end{array}$ & June '09 \\
\hline $09-22$ & $\begin{array}{l}\text { T. Aiki } \\
\text { M.J.H. Anthonissen } \\
\text { A. Muntean }\end{array}$ & $\begin{array}{l}\text { On a one-dimensional shape- } \\
\text { memory alloy model in its } \\
\text { fast-temperature-activation } \\
\text { limit }\end{array}$ & June '09 \\
\hline $09-23$ & J. Portegies & $\begin{array}{l}\text { Efimov trimers in a harmonic } \\
\text { potential }\end{array}$ & July '09 \\
\hline $09-24$ & $\begin{array}{l}\text { M.J.H. Anthonissen } \\
\text { J.H.M. ten Thije } \\
\text { Boonkkamp }\end{array}$ & $\begin{array}{l}\text { A compact high order finite } \\
\text { volume scheme for } \\
\text { advection-diffusion-reaction } \\
\text { equations }\end{array}$ & August '09 \\
\hline
\end{tabular}

